\title{
Influence of Rainfall on Band Characteristics of the Ka Band
}

\author{
Zheng Liu , Huihui Zou , Fuan Su \\ China Satellite Tracking and Controlling Department, Jiangyin 214431, China \\ liuzheng354@qq.com
}

\begin{abstract}
Keywords: Characteristics; Rainfall; Ka Band
Abstract. At present, most of the measurement and control communication services in the aerospace field utilize the $\mathrm{S}$ and $\mathrm{C}$ frequency bands. The usage of the higher $\mathrm{X}$ and $\mathrm{Ku}$ frequency channels is also increasing. However, as the frequency band resources become more and more crowded, the Ka frequency band necessarily becomes the future development of space communications measurement and control trends due to its wide working frequency channel, large communication capacity, strong anti-interference ability and other advantages. At the same time, the development of the Ka frequency band is also confronted with many problems, including the limited production level of high-frequency devices, the difficulty of narrow beam tracking, the attenuation of rain and the attenuation of atmosphere and other problems. This article mainly analyzes the influence of rainfall attenuation on characteristics of the Ka frequency band.
\end{abstract}

\section{Introduction}

The Ka-band microwave's wavelength is at the millimeter level. It develops rapidly due to its short wavelength and wide band. In addition, there are several important reasons. The maturity of the microwave system has made that the low-frequency microwave spectrum is very crowded. Besides, the available spectrum of high-frequency band is wider with larger capacity. Ka-band antenna easy to meet the demand of the narrow beam with high gains. Therefore, resolution is higher and anti-interference ability is stronger. Increases in the Doppler shift can also increase the sensitivity of speeds. At the same time, spacecrafts in the atmosphere at high speed can form a plasma layer, making the so-called black-barrier area. It leads to serious deterioration of the signal strength when the radio signal passes. And it will seriously affect the signal phase characteristics. Studies have shown that the lower the operating frequency, the higher the degree of influence. The Ka band has the ability to break through the black barrier area. Therefore, it will have a good future in the field of aerospace measurement and control.

\section{Research significance of rainfall's influence}

The development of the Ka band has become an important development direction for aerospace measurement and control communications. Researchers are faced with many difficulties, such as the limited production level of high-frequency devices, the difficulty of narrow beam tracking, the attenuation of rain and the attenuation of atmosphere and other problems. However, with the development of multi-beam guidance technology, the emergence of high-dynamic and high-precision servo tracking technology has initially solved the difficulty of high dynamic angle tracking of narrow beams. In the meantime, the improvement of the Ka band power synthesis amplification technology has further accelerated the Ka band's development in the field of aerospace measurement and control communications. Currently, the key to be further overcome is the influence of rain attenuation on the channel characteristics. According to the theoretical analysis, if the frequency band is higher, the wavelength will be closer to or below the size of the raindrop. The greater the attenuation is, the more unstable the signal polarity is. The greater the change in phase value will seriously affect the accuracy of angle tracking. It can even lose the target, leading to the failure of the most important tele-control of spacecraft. 


\section{Influence of rainfall on the Ka band}

After the working frequency band is increased to the Ka frequency band, it will cause problems of rainfall attenuation, airborne propagation loss and increases in noise. Rain attenuation is the main factor. It is caused by attenuation caused by the radio wave absorption, refraction and reflection and scattering due to weather factors such as rain and snow. The ionosphere can absorb the radio, changing the polarization characteristics. According to statistics, in the Ka band, the general rainfall or snowfall will be reduced by about $10 \mathrm{~dB}$. However, in rainy or snowy days, the attenuation will even reach more than $20 \mathrm{~dB}$. Therefore, the rainfall attenuation becomes an important factor.

In the field of traditional space flight measurement and control communication, the signal level received on the downlink is reduced, which will make the ground equipment unable to track the spacecraft normally. At the same time, the signal-to-noise ratio of the spacecraft declines, and the bit error rate will increase, which affects the accuracy and the reduction of the system of communication transmission rate, making the band to enhance the advantages cannot be reflected. For the uplink, the signal strength of the transmitted signal attenuated by rain and arriving at the spacecraft may not trigger the transponder, causing important remote control commands to fail to be transmitted to the spacecraft, which could become a serious accident.

In summary, the benefits of lower rainfall attenuation and higher band will cancel each other out. In most climates, the Ka band is better than the lower band, but the equipment will not work normally under heavy rain or heavy snow, Work in all weather conditions.

\section{Case Analysis of the influence of rainfall}

In order to further analyze the characteristics of rain attenuation, it needs to analyze results of multiple standard calibration at different locations, different seasons and different weather in the same set of equipment. Since the Ka band system has a large number of equipment in an outdoor environment, it is not recommended to use this equipment with charge in extreme weather. In addition, we cannot do a precise quantitative analysis of rainfall statistics, we can only do a qualitative analysis through the accumulation of equipment in the calibration of the data. Through the long-term accumulation of calibration results, others are recorded under basically the same environment except the weather. Results are shown in the following table.

Table 1 The channel characteristics' statistical table under different conditions

\begin{tabular}{|c|c|c|c|c|c|}
\hline Date & equipment & weather & temperature $\left({ }^{\circ} \mathrm{C}\right)$ & $\mathrm{AGC}(\mathrm{V})$ & Phase change $\left(^{\circ}\right)$ \\
\hline 2017/5/23 19:18 & FM & Moderate rain & 25.9 & 1.6 & -3.2 \\
\hline 2017/5/31 21:45 & FM & sunny & 22.9 & 2.4 & 0 \\
\hline 2017/6/6 09:54 & FM & Light rain & 15.8 & 2.1 & 11 \\
\hline $2017 / 8 / 8$ 15:30 & FM & Heavy rain & 27 & 1.2 & -59 \\
\hline
\end{tabular}

According to the theoretical analysis and the accumulated data analysis, the magnitude of rainfall attenuation is related to the amount of rainfall and the size of the raindrops. Meanwhile, the magnitude of the rainfall attenuation is also related to the frequency. The frequency and the wavelength are inversely related to each other. However, the relationship between wavelength and raindrop's diameter can affect the amount of attenuation. At the same time, the temperature and humidity also affect the channel characteristics of Ka. Therefore, other factors need to be distinguished when rainfall attenuation is analyzed. The effect of rainfall on the phase-frequency characteristics of signals is because that the shape of raindrops can change the polarization characteristics the radio waves. It makes the phases changes dramatically when it rains. The tracking of the spacecraft will also be affected. 


\section{Analysis of anti-rain attenuation measures}

The characteristics of the Ka band waves are greatly affected by rainfall. Once it is bad weather, the communication of the spacecraft will be hindered. Therefore, some solutions are needed to deal with extreme weather. In general, the Ka device have a corresponding channel margin when it is designed. However, if the margin is fixed, it will make its coping ability weak. It is easy to make the signal level below the limit or saturation, reducing the system's robustness. However, if it is designed to be dynamically adjusted, its compensation accuracy is more difficult to control. The spacecraft's design is complex. Therefore, we need to estimate the rainfall attenuation's value. And then we should adjust the uplink and downlink based on the estimated value. The design of the transponder on the spacecraft should be light and easy to control, and the answering machine's adjustment needs to issue the uplink remote control command through the ground equipment. The rainfall attenuation may invalidate instructions that are received by the transponder. Therefore, the adjustment is mainly considered from the ground equipment. The attenuation's control range should be increased at the receiving end. The upper limit of transmit power should be increased at the transmitter. At present, the anti-rain attenuation technology mainly include the power control technology, adaptive technology, signal processing technology. In order to better improve the practicability of the Ka system, it is necessary to use as many different methods as possible to deal with it in the operable range to gain the best result.

At present, the popular Ka-band rainfall attenuation model is the ITU-R model. The Ka band spacecraft is needed to test it. Currently, Ka-band satellites have not been widely used in our country. There is no condition to measure Ka-band rain attenuation. Therefore, the method of estimating rainfall attenuation can only rely on the beacon. To determine the relationship between rain attenuation and the length of the rain area, the beacon signal's intensity in good weather should be firstly calibrated. The beacon is generally fixed at different distances. Under different far fields, the signal strength is measured after the attenuation in the rainy weather. The attenuation value of different distances at a fixed frequency can be obtained. Similarly, in order to determine the relationship between rainfall attenuation and frequency, under a fixed distance beacon, the rainfall attenuation value under different frequencies should be measured respectively. Through the accumulation of long-term data, the frequency and distance can be converted, which aims to get faster estimation of rainfall attenuation values at different frequencies and distances. If the frequency conversion can reach the Ka band, it will be more convenient to calibrate the rainfall attenuation values through in-orbit spacecraft. Then through the conversion method, the more accurate Ka-band rainfall attenuation value is obtained.

Because the rainfall attenuation value is a process with relatively slow changes, the size and time of the residual capacity can be adjusted by real-time to meet the requirement of rain attenuation. Through the rainfall attenuation estimation of the downlink, combined with the experience of frequency conversion, the uplink rain attenuation can be estimated at the same time to do the dynamic compensation of the uplink. During the adjustment process, the adjustment threshold and the time should be concerned to avoid overcompensation. Anti-rainfall attenuation usually needs to leave enough adjustable margin of the equipment, different uplink and downlink polarization will affect rainfall attenuation. Meanwhile, under different rainfall attenuation conditions, different information transfer rates can reduce the deviation of rain attenuation adjustment.

\section{Conclusions}

Based on the theoretical study of rainfall attenuation in the Ka band both at home and abroad, this article puts forwards a method for estimating rainfall attenuation under existing conditions with $\mathrm{Ka}$ equipment. The related factors affecting rainfall attenuation are verified by existing equipment, mainly including frequency, temperature, rainfall and the raindrop size and so on. Based on beacon calibration in far field, a scheme of estimating rainfall attenuation in uplink and downlink in combination with 
frequency conversion is proposed. It provides data support for rain attenuation regulation in dynamic environment. Follow-up work will further accumulate and improve the influence of rainfall attenuation on this equipment at different rainfall intensity and different frequencies, continuously improving the rain-resistant capacity of the device.

\section{References}

[1] Wang Cheng-yuan. Measurement and Frequency Conversion of Rain Attenuation for Satellite Communication Link [J]. Wireless Comunication Technology, 2010,19(2):37-40.

[2] Yu Miao. Mixed Rain Attenuation Compensation Algorithm of Ka-Band Satellite Communication System [J]. Journal of Jilin University, 2015, 33(1):33-38.

[3] Liu Jiaxing. Spacecraft TT\&C and Information Transmission Technology[M]. Beijing: National Defend Industry Press, 2011.

[4] Wang Hongfeng. Short-time rain attenuation prediction for Ka-band satellite communication system based on numerical weather prediction[J]. Chinese Space Science and Technology,2017,37(1):82-88. 